Introduction: Improving public access and training for epinephrine auto-injectors (EAIs) can reduce time to initial treatment in anaphylaxis. Effective use of EAIs by the public requires bystanders to respond in a timely and proficient manner. We wished to examine optimal methods for effective training and skill retention for public use of EAIs. Methods: In this prospective, stratified randomized study, 154 participants at 15 sites receiving installation of public EAIs were randomized to one of three experimental education interventions: A) didactic poster (POS) teaching; B) poster with video teaching (VID), and C) Poster, video, and simulation training (SIM). Participants were tested by participation in a standardized simulated anaphylaxis scenario at 0-months, immediately following training, and again at follow-up at 3 months. Participants responses were videoed and assessed by blinded raters. Patient recorded experience measures (PREMs) assessed participantpatient interaction for every scenario. Data that was non-normally distributed was analyzed using non-parametric testing (Kruskall-WallisRank Sum-Test). Results: Initial analysis showed differences between group baseline characteristics for age and first aid training; with a multivariable analysis providing the effect size of these differences. PREM data and video assessment data were not normally distributed. Analysis of PREM data revealed significantly higher scores in the SIM group at 0 -months $($ median $=6.5, \mathrm{IQR}=5 ; \mathrm{p}=0.05$ ) and 3 -months (median $=5, \mathrm{IQR}=3 ; \mathrm{p}<0.01$ ), compared to those groups that did not receive SIM. Video assessment performance scores show trends in higher skills and knowledge retention for SIM participants at 3-months; full data analysis will be performed at a later date. Final video assessment analysis will involve a weighted scoring system, using a consensus process, and an inter-rater agreement analysis. Conclusion: Simulation training improves interaction, essential skills, and retention of knowledge in simulated anaphylaxis response with public EAIs compared to non-simulation-based training.

Keywords: anaphylaxis, simulation training, epinephrine auto-injectors

\section{P038 \\ Emergency medicine interest group: evaluation of a student led organization at Memorial University}

C. Dunne, BSc, D. Hansen, BScN, M. Parsons, MD, Memorial University, St. John's, NL

Introduction: Interest groups have become increasingly popular as students explore potential career paths earlier in their undergraduate experience. Emergency medicine (EM) has grown as a specialty and the match has become quite competitive. Attractive features of EM cited by learners (diversity, procedural skills and flexible schedule) appeal broadly to the undergraduate population. Learners at Memorial University recognized this leadership opportunity and worked with faculty to reach this wide target audience through a streamlined iterative evaluation of their EM Interest Group (EMIG). Methods: The local EMIG was formed in 2010. Yearly, EMIG executive work with outgoing members using prior experiences, contacts and best practices to facilitate handover and progress. From 2015 to present, 305 surveys were collected, giving an $81.9 \%$ response rate. $59.7 \%$ of respondents were first year students, and $40.3 \%$ were second year. The survey consisted of Likert scale and open-response questions. The Likert scale questions yielded favorable responses. 304 students $(99.6 \%)$ felt presenters were knowledgeable, 301 (98.6\%) would recommend the sessions to others and $301(98.6 \%)$ were satisfied they attended. Surprisingly, 133 students (43.6\%) said they were not interested in Emergency Medicine, likely attending due to the appeal of session topics and transferrable of EM skills. $232(76.0 \%)$ stated that attendance did increase their interest in EM. Top responses for aspects of EM most interesting to them included: ability to find a work/life balance, ability to work urban or rural, variety of cases seen, and the non-routine shifts. Results: Survey feedback is used to inform refinement of the content, delivery and format of EMIG activities, delivered by EM faculty. Hands-on sessions (e.g. suturing \& airway management) have been popular. Informational sessions, on specific medical topics (ECG, resuscitation cases) or broader topics (EM streams) have also been very well received. Inclusion of all interested students, particularly large numbers for hands-on sessions, has presented challenges. Beyond current survey results, it will be interesting to consider if EMIG participation translates to learning or behavioral changes relevant to later clinical encounters; a question that will be difficult to quantify. Conclusion: The EM interest group is one of the most active at Memorial University. Survey results indicate that participants enjoy the EMIG session content and the structured iterative approach used by the group has been successful in maintaining an effective student led organization.

Keywords: innovations in emergency medicine education, student lead interest group, survey evaluation

\section{P039}

Application of the Delphi method to refine key components in the iterative development of a mobile tele-simulation unit (MTU)

C. Dunne, BSc, J. Jewer, PhD, M. Parsons, MD, Memorial University, St. John's, NL

Introduction: Safe and efficient provision of quality healthcare requires maintenance of knowledge and skills relevant to daily practice. This is particularly relevant in rural and remote locations where high-acuity low-occurrence procedures and clinical scenarios present even less frequently. Simulation based training is widely used to supplement clinical exposure and practice but effective delivery of this approach to the rural/ remote practitioner must address barriers of time, cost and geographical separation. Mobile tele-simulation is an innovative approach that may help in bridging the gap through delivery of effective mentoring using telemedicine technologies and tailored educational content. Methods: To help direct the iterative design cycle for the mobile tele-simulation unit, input from potential future users was felt to be essential. The Delphi method was employed to reach consensus among study participants on four key questions: 1) What applications would the MTU be best suited for?, 2) What technical requirements and teaching tools would be needed to make the MTU successful?, 3) Which fields, besides EM/medical education, may benefit from partnerships with the final MTU?, 4) What research studies could be developed using the MTU? It was decided in advance that two rounds would be the maximum due to time constraints of the larger MTU projects. The first questionnaire focused on demographics and the four questions above. Independent reviewers analyzed, compiled and compared responses. Participants were sent the updated list, asked to confirm their responses and then to rank the responses highest to lowest priority. Results: Fifteen of 17 first round participants completed the questions, giving an $88.2 \%$ response rate. All shared a simulation background. $66 \%$ were physicians, $13.3 \%$ medical students, and $20 \%$ staff at Memorials Simulation Center. $66 \%$ had been involved with simulation-based education less than 5 years, and the others greater than 5 year. 13 of 15 $(86.7 \%)$ responded in round 2 . Consensus was not reached statistically using Kendalls W test for each of the four questions. However, there were several responses that showed higher median ranks than the others under each question. Application use: rural healthcare training, and medical professional training Technical factors: reliable learner-mentor connection, and competent technical support Non-technical factors: knowledgeable mentor and content relevant to locations practice, Research studies: training needs assessment from rural sites, and 